\section{§18. Fluid Simulation on Pellet Ablation with Atomic Process}

Ishizaki, R., Parks, P.B. (General Atomics)

Refueling is one of essential methods in order to control plasma density and sustain steady state plasmas. A gas puffing has been successful for building and sustaining a plasma density in an experimental system of past generation. However, in a large scale experimental system, e.g., LHD, the plasma sources induced by the gas puffing are strongly localized near the plasma surface. Then, a pellet injection is placed as a fundamental tool and has been mainly used to obtain a high density plasma and control a density profile [1].

When a pellet consisting of solid hydrogen is heated by an energy flux, it changes to vapor and subsequently to plasma through atomic process. In the present work, the ablation cloud consists of molecules, atoms, ions and electrons. Effects of atomic physics on the ablation process are considered. It is assumed that the species have same velocity and same temperature due to the high collision and charge exchange. An electromagnetic force is ignored for simplicity. In addition, the domain of the simulation includes solid and gas regions by using the Cubic-Interpolated Psudoparticle (CIP) method that can calculate simultaneously incompressible and compressible regions [2]. The heat flux is carried by the incident electrons which the distribution of energy is taken as Maxwellian.

The code is time dependent, starting from an initial condition of a bare pellet in a bulk plasma of specified electron temperature $\left(T_{e \infty}\right)$ and number density $\left(n_{e \infty}\right)$. Figure 1 shows special profiles obtained by running up to $8 \mu$ sec. in 1D spherical symmetry where $p_{s}, T_{s}$ and $r_{s}$ are the pressure, temperature and radius at the sonic point,

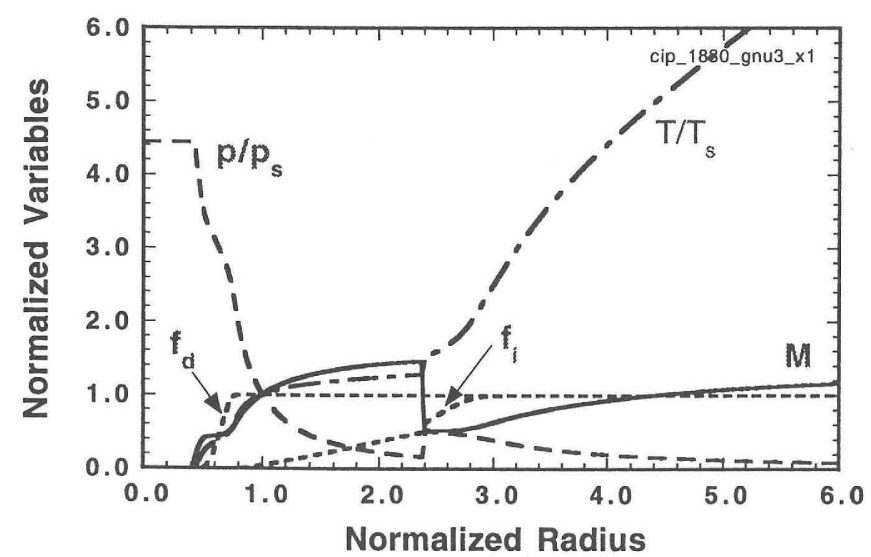

Fig. 1: Normalized fluid profiles at $8 \mu \mathrm{sec}$. A dashed, dotdashed and solid lines show $M, p / p_{s}, T / T_{s}$ as functions of $r / r_{s}$, respectively. Dot lines show $f_{d}$ and $f_{i}$.

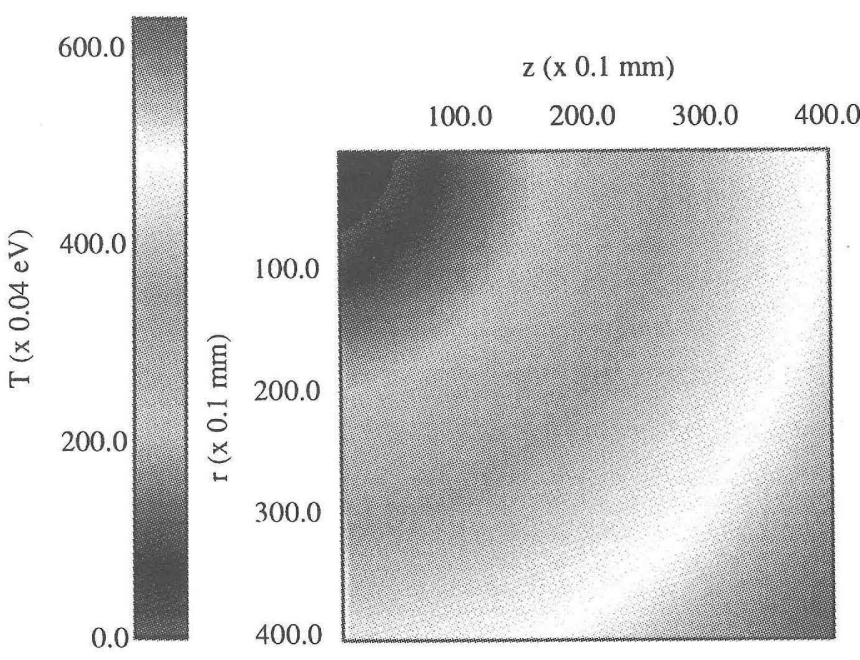

Fig. 2: Temperature contour on $r-z$ plane at $8 \mu$ sec.

respectively. The parameters used are the pellet radius $r_{p}=2 \mathrm{~mm}$, electron temperature $T_{e \infty}=2 \mathrm{keV}$ and number density $n_{e \infty}=10^{20} \mathrm{~m}^{-3}$ in the bulk plasma. $M$ has a jump structure around $r / r_{p} \simeq 2.4$ where $M$ on the pellet side is greater than unity and $M$ on the other side out is less than unity. Since the flow goes from the pellet surface to the bulk plasma, an incident flow becomes supersonic and a transmitted flow becomes subsonic in a reference frame moving with this structure. In addition, ionization is dominant in the region as shown by $f_{i}$. Then, this structure is clear to be a shock wave driven by ionization.

Next we evaluate effects of the heat flux propagating into the cloud along the magnetic field $(z)$. The geometry of the code is cylindrical with symmetry assumed about the magnetic field line that passes through the center of the pellet. Figure 2 shows a temperature contour at $8 \mu \mathrm{sec}$. where a black region corresponds to the pellet. The parameters used are $r_{p}=5 \mathrm{~mm}, T_{e \infty}=4 \mathrm{keV}$ and $n_{e \infty}=10^{20} \mathrm{~m}^{-3}$. It is found that the pellet radius in the $r$ direction becomes longer than one in the $z$ direction. This comes from nonuniform pressure on the pellet surface. Almost energy of the heat flux through the pole of the pellet is deposited around the pellet surface. On the contrary, a part of the heat flux energy through the equator is deposited around the pellet and another goes through the pellet. Then, since the pressure at the pole is greater than one at the equator of the pellet, the pellet makes deformation. Effects of atomic process with a shock wave will be evaluated in the pellet deformation.

\section{REFERENCES}

[1] L. R. Baylor. et al., Nucl. Fusion 32, 2177 (1992).

[2] T. Yabe and P. Y. Wang, J. Phys. Soc. Jpn 60, 2105 (1991). 